\title{
A Refined Hilbert-Huang Transform With Applications to Interarea Oscillation Monitoring
}

\author{
Dina Shona Laila, Member, IEEE, Arturo Roman Messina, Senior Member, IEEE, and \\ Bikash C. Pal, Senior Member, IEEE
}

\begin{abstract}
This paper focuses on the refinement of standard Hilbert-Huang transform (HHT) technique to accurately characterize time varying, multicomponents interarea oscillations. Several improved masking techniques for empirical mode decomposition (EMD) and a local Hilbert transformer are proposed and a number of issues regarding their use and interpretation are identified. Simulated response data from a complex power system model are used to assess the efficacy of the proposed techniques for capturing the temporal evolution of critical system modes. It is shown that the combination of the proposed methods result in superior frequency and temporal resolution than other approaches for analyzing complicated nonstationary oscillations.
\end{abstract}

Index Terms-Convolution filter, empirical mode decomposition, Hilbert-Huang transform, interarea oscillation, masking.

\section{INTRODUCTION}

$\mathbf{T}$ RANSIENT response of power systems typically displays nonstationary characteristics [1]. Extracting and quantifying temporal modal behavior from the observed oscillations present a significant challenge due to the nature of switching events and other control actions that may take place over the observation period [2]-[8].

Modal analysis is one of the most effective techniques to extract modal information from power systems models [9]-[11]. However unfortunately, oscillatory processes may exhibit nonlinear behavior and in many cases linear models are not sufficient to capture time-varying features associated with switching and control actions. Several other complementary techniques based on ringdown analysis to system perturbations and MIMO state-space identification techniques have been successfully applied to analyze wide-area oscillatory dynamics [3], [4], [8], [12], [13]. Fourier-based analysis tools have also been used for off line studies of power system dynamics [14], [15]. These techniques, however, rely on the assumption of linearity and assume that the data are strictly periodic or stationary in time which limits their applicability to real problems. In addition, Fourier spectrum defines uniform harmonic components glob-

Manuscript received May 30, 2008; revised October 07, 2008. First published April 10, 2009; current version published April 22, 2009. This work ws supported by the EPSRC Research Grant EP/E3032435/1, in collaboration with ABB, Switzerland, and National Grid, U.K. Paper TPWRS-00427-2008.

D. S. Laila and B. C. Pal are with the Electrical and Electronic Engineering Department, Imperial College, London SW7 2AZ, U.K. (e-mail: d.laila@imperial.ac.uk; b.pal@imperial.ac.uk).

A. R. Messina is with the Graduate Studies Program, Cinvestav, Guadalajara 45010, Mexico (e-mail: aroman@gdl.cinvestav.mx).

Digital Object Identifier 10.1109/TPWRS.2009.2016478 ally and therefore needs many additional harmonic components to simulate nonstationary data.

Other efforts include the use of ARMA block-processing techniques to estimate stationary low-frequency modes from measured ambient power system data [16] and the development of mode meter block-processing algorithms for an automated dynamic stability assessment [17]-[19]. Examples of these approaches include the modified Yule-Walker method, the extended modified Yule-Walker with spectral analysis and sub-space system identification methods.

Despite these advances, modal characterization under proper analysis remains a challenge due to the complexity of the driving system processes operating on various temporal scales. Recently, nonlinear and nonstationary analysis techniques based on the Hilbert-Huang transform (HHT) [20] have been used to analyze data from nonlinear and nonstationary processes [6], [21], [22]. The method has been applied to many important problems in various fields including medical [23], geophysics [24], and power engineering [25].

The cornerstone to the whole HHT procedure is the empirical mode decomposition (EMD) that separates a signal into a series of amplitude-as well as frequency - modulated signal components [20], [26]. Extracting these signal components from a data set, however, is very challenging and may involve various complications including mode mixing and the generation of spurious information for various types of signals. This may obscure physical interpretation of the system behavior, especially when the observed oscillations exhibit closely spaced modes. Other issues are the end effects associated with the computation of the Hilbert transform and the smoothness of the representations. All these issues have motivated considerable recent research into developing methodologies to improve the HHT.

To address the problem of mode mixing, EMD with masking technique was introduced in [27] and [28]. It also solves the problem of intermittency that prevents the effectiveness of EMD. Based on this masking technique, in [29] and [30], a systematic procedure for constructing the masking signals is proposed. The ability of these approaches to analyze power quality signals with relatively high frequency was discussed in [29]. While these approaches are found to work well on various types of signals, simulation results show that they may fail to provide characterization of more complicated system behavior, especially for signals with low frequency components, low magnitude or narrow frequency range.

This research investigates several extensions to the HHT technique. Improvements to both the masking technique and the computation of Hilbert transformers are proposed, and a number 
of issues within their use and interpretation are identified. The efficacy of the method to separate closely spaced modal components is demonstrated on both synthetic and transient stability data. It is shown that the method produces a physically motivated basis suitable for analysis of general nonlinear and nonstationary signals, particularly for interarea oscillation monitoring and analysis.

\section{PRELIMINARIES}

In an effort to make the paper reasonably self-contained, the standard algorithm of the HHT and its components, the EMD technique, Hilbert transform and the damping computation, are briefly reviewed. Our development follows the development of Huang [20], to which we refer the readers for more details.

\section{A. Empirical Mode Decomposition Method}

The EMD method provides an analytical basis for the decomposition of a signal $x(t)$ into a set of basis functions, called intrinsic mode functions (IMFs). An IMF is defined as a signal that satisfies the following criteria.

1) Over the entire time series the number of extrema and the number of zero-crossings differ by, at most, one, i.e., an essentially oscillatory process.

2) At any point the mean value of the envelope defined by the local maxima and the envelope defined by the local minima is zero.

The basic EMD method adopted to extract the IMFs essentially consists of a three-step procedure called sifting [6]. The goal is to subtract away the large-scale features of the signal repeatedly until only the fine-scale features remain. A signal $x(t)$ is thus divided into the fine-scale details $c(t)$ and the residue $r(t)$, hence $x(t)=c(t)+r(t)$. The components contained in the fine-scale details are the IMFs.

The standard EMD process can be summarized as follows.

S1. Given the original signal $x(t)$, set $r_{o}(t)=x(t), j=1$.

S2. Extract the $j$ th IMF using the sifting procedure:

a) Set $i=1$ and $h_{i-1}(t)=r_{j-1}(t)$.

b) Identify the successive local minima and the local maxima for $h_{i-1}(t)$. The time spacing between successive maxima is defined to be the time scale of these successive maxima.

c) Interpolate the local minima and the local maxima with a cubic spline to form an upper $e_{\max _{i-1}}(t)$ and lower $e_{\min _{i-1}}(t)$ envelope for the whole data span.

d) Compute the instantaneous mean of the envelopes, $m_{i-1}(t)=\left(e_{\min _{i-1}}(t)+e_{\max _{i-1}}(t)\right) / 2$; and determine a new estimate $h_{i}(t)=h_{i-1}(t)-m_{i-1}(t)$, such that $e_{\min _{i-1}}(t) \leq h_{i}(t) \leq e_{\max _{i-1}}(t)$ for all $t$. Set $i=i+1$.

e) Repeat steps $2 \mathrm{~b}-2 \mathrm{~d}$ until $h_{i}(t)$ satisfies a set of predetermined stopping criteria [follows the criteria 1) and 2) of an IMF]. Then set $c_{j}(t)=h_{i}(t)$.

S3. Obtain an improved residue $r_{j}(t)=r_{j-1}(t)-c_{j}(t)$. Set $j=j+1$. Repeat step S2 until the number of extrema in $r_{j}(t)$ is less than 2 .

This approach allows elimination of low amplitude riding waves in the time series and eliminates asymmetries with respect to the local mean, i.e., it makes the wave profile more symmetric. At the end of this process, the EMD yields the following decomposition of the signal $x(t)$ :

$$
\begin{aligned}
x(t) & =\sum_{j=1}^{n} c_{j}(t)+r_{n}(t) \\
& =\sum_{i=1}^{q} c_{i}(t)+\sum_{k=q+1}^{p} c_{k}(t)+\sum_{l=p+1}^{n} c_{l}(t)+r_{n}(t)
\end{aligned}
$$

where $q<p<n, c_{i}(t), i=1, \ldots q$ contain high frequency noise components, $c_{k}(t), k=q+1, \ldots p$ contain the physical behavior of interest and the remaining terms $c_{l}(t), l=$ $p+1, \ldots n$ and $r_{n}(t)$ contain less relevant, nonsinusoidal characteristics. Note that in some applications where the noise does not involve or has been removed through filtering, the first $q$ components may not exist.

\section{B. Hilbert Transform}

Given a real signal $x(t)$, its complex representation is

$$
z(t)=x(t)+i x_{H}(t)
$$

where $x_{H}(t)$ is the Hilbert transform of $x(t)$, given by

$$
x_{H}(t)=\frac{1}{\pi} P \int_{-\infty}^{+\infty} \frac{x(s)}{t-s} d s
$$

with $P$ the Cauchy principal value of the integral. Equation (2) can be rewritten in an exponential form as

$$
z(t)=A(t) e^{i \psi(t)}
$$

where

$$
\begin{aligned}
A(t) & =\sqrt{x(t)^{2}+x_{H}(t)^{2}} \\
\psi(t) & =\arctan \frac{x_{H}(t)}{x(t)} .
\end{aligned}
$$

The time derivative of (4) is

$$
\dot{z}(t)=A(t) e^{i \psi(t)}(i \omega(t))+e^{i \psi(t)} \dot{A}(t)
$$

where $\omega(t)$ is the instantaneous angular frequency, which by definition is the time derivative of the instantaneous angle

$$
\omega(t)=\dot{\psi}(t)=\frac{d}{d t} \arctan \frac{x_{H}(t)}{x(t)} .
$$

Hence, the instantaneous frequency can be defined as $f(t)=$ $(\omega(t)) /(2 \pi)$, and using (4) and (7), it can be computed as

$$
f(t)=\frac{1}{2 \pi} \operatorname{Im}\left(\frac{\dot{z}(t)}{z(t)}\right)=\frac{x(t) \dot{x}_{H}(t)-x_{H}(t) \dot{x}(t)}{2 \pi\left(x^{2}(t)+x_{H}^{2}(t)\right)} .
$$

\section{Damping Ratio $(\zeta)$ Estimates}

The knowledge about the instantaneous magnitude and instantaneous frequency of a signal allows us to further compute the instantaneous damping of the signal. Damping characterization is another useful alternative to the analysis of local behavior of the oscillation. Consider the signal (4). We can rewrite the 


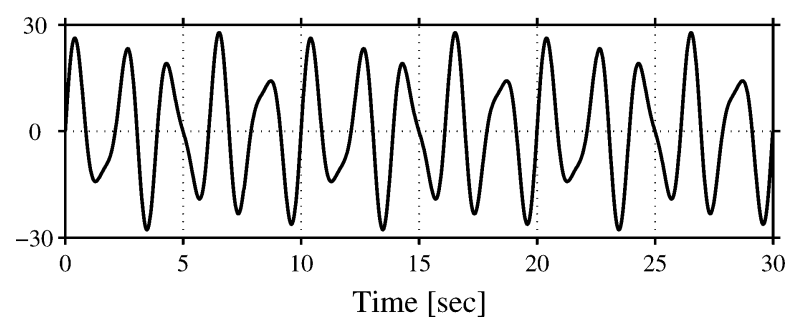

Fig. 1. Two-component synthetic signal (15).

signal as [31]

$$
z(t)=A(t) e^{i \psi(t)}=\Lambda(t) e^{-\theta(t)+i \psi(t)} .
$$

Then the time dependent decay function can be modeled as

$$
\theta(t)=-\int_{0}^{t} \alpha(t) d t
$$

Moreover, using (4) and (7), we obtain

$$
\frac{\dot{z}(t)}{z(t)}=\left[\left(-\alpha(t)+\frac{\dot{\Lambda}(t)}{\Lambda(t)}\right)+i \omega(t)\right] .
$$

Noting that

$$
\operatorname{Re}\left(\frac{\dot{z}(t)}{z(t)}\right)=\frac{\dot{A}(t)}{A(t)}
$$

we have

$$
\alpha(t)=-\frac{d \theta(t)}{d t}=-\left[\frac{\dot{A}(t)}{A(t)}-\frac{\dot{\Lambda}(t)}{\Lambda(t)}\right] .
$$

We emphasize that (14) is a generalization to modal analysis of the notion of damping for nonstationary signals. The computation of damping ratio $\zeta$ from local information in (14) depends on the fast and accurate estimation of the physically meaningful instantaneous magnitude $A(t)$ which is given by (5). Also, approximating the signal with an exponential signal, we should be able to find a constant or at least a slowly varying $\Lambda(t)$, such that $\dot{\Lambda}(t) \approx 0$. Therefore, to make sure that the computation is amenable to temporal modal analysis, a physically motivated basis for the data is requited. To obtain the damping ratio estimate of a range of signal, the average (mean value) of the instantaneous damping is computed.

Remark 2.1: In HHT technique, Hilbert transform is applied to each IMF to compute its instantaneous frequency, instantaneous magnitude, as well as instantaneous damping. As instantaneous frequency is best defined for mono-frequency signal, i.e., signal that contains only one (dominant) frequency, it makes sense to expect each IMF to be mono-frequency. However, as pointed out earlier, the IMFs may contain a mixture of frequencies (frequency modulation) and are difficult to interpret in terms of conventional modal analysis. This has motivated the need for demodulation techniques that extract from each IMF the dominant interacting frequencies.

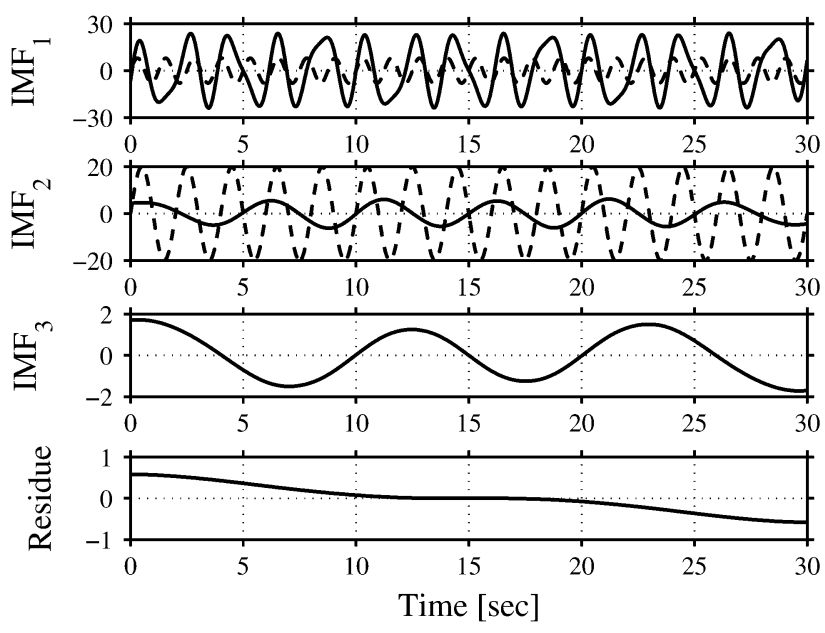

Fig. 2. IMFs of signal (15) obtained from standard EMD. The dashed grey lines are the 0.8 and $0.5 \mathrm{~Hz}$ components of (15).

\section{MASKING TeChNiques To IMPROVE EMPIRICAL MODE DECOMPOSITION}

This section discusses the refinement of the conventional EMD method to study the oscillatory dynamics, particularly that involve the identification of frequency within the range on 0.1 to $1 \mathrm{~Hz}$ which is the typical range of power systems interarea modes. First, a synthetic example is introduced to examine conditions under which the standard HHT and the conventional masking technique may fail. Then, various algorithms to refine the existing HHT are proposed.

\section{A. When the Standard EMD Does Not Work}

Consider a two-component signal, $x(t)$

$$
x(t)=8 \sin (1.6 \pi t)+20 \sin (\pi t) .
$$

The time evolution of this testing signal is shown in Fig. 1. The clear feature of signal (15) is that it consists of low frequency components and the magnitude of the higher frequency component is significantly lower than that of the lower frequency component.

The standard EMD [32] is applied to the signal (15). Fig. 2 shows the IMF components extracted using this procedure. The dashed plots with the first two IMFs are the 0.8 and $0.5 \mathrm{~Hz}$ components of the signal (15). Quite contrary to what is expected, $\mathrm{IMF}_{1}$ and $\mathrm{IMF}_{2}$ do not imitate the two sinusoidal components of signal. Moreover, it is obvious that $\mathrm{IMF}_{1}$ is not a mono-frequency signal, but instead it exhibits mode mixing, making little sense to expect useful physical interpretation through the application of Hilbert analysis. The discrepancies between the decomposition result and the components of the signal propagate to other IMFs making the overall extraction of temporal behavior difficult.

In an attempt to improve the performance and effectiveness of EMD, the use of masking signals is introduced in [27] and [28]. The technique aims at solving the problem of mode mixing and ambiguity that occur when two or more frequencies are not well separated. More in-depth discussion about the background and technicalities of this technique is presented in [28]. Further development of EMD with masking is proposed in [29]. 


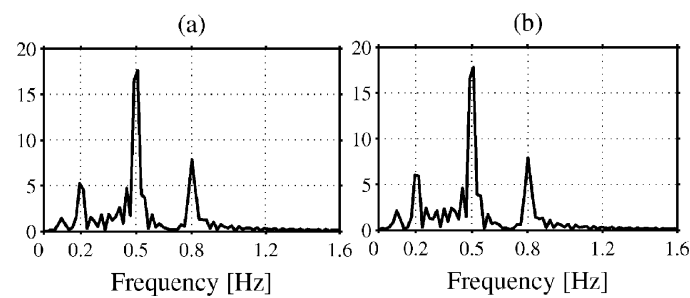

Fig. 3. Fourier spectrum of the first IMFs of signal (15) from (a) standard EMD and (b) EMD with masking [29].

Although this technique has proved effective in analyzing a large variety of signals, some limitations arise in the study of composite oscillations involving low-frequency components. To investigate further these limitations, we applied the masking technique from [29] to the signal (15). Fig. 3 that compares the spectra of the first IMF obtained using conventional EMD with that of the approach in [29] does not show any improvement. As the frequency components of the signal, in this case are 0.8 and $0.5 \mathrm{~Hz}$, are very low and consequently the $0.3 \mathrm{~Hz}$ difference between them is very small, this existing masking technique becomes ineffective in separating these components. Techniques to effectively identify and isolate the individual frequency components are discussed in the following subsection.

\section{B. EMD Method With FFT-Based Masking Technique}

It is comprehensible from the discussion and examples in Section III-A that issues affecting the effectiveness of standard EMD and the existing masking techniques are

- the signal consists of low frequency components ${ }^{1}$;

- the magnitude of the highest frequency component is much lower than others, particularly the second component, which is directly next to it in the Fourier spectrum;

- the frequency components are high enough, but they are relatively close to each other.

Based on the above considerations, we proposed the use of a unified masking signal that in some sense refines the results of [29] and at the same time generalizes the results of [27] and [28]. The algorithm of the refined EMD, named as R-EMD, is described as follows.

R1. Perform FFT on the original signal $x(t)$ to estimate the frequency components $f_{1}, f_{2}, \ldots f_{n}$, with $f_{1}>f_{2}>$ $\cdots>f_{n}$. These captured frequencies are the stationary equivalence of the possibly time varying frequency components of the signal $x(t)$.

R2. Construct the masking signals mask 1 , mask $_{2}, \cdots$, mask $_{n-1}$ using the following sinusoidal signals:

$$
\operatorname{mask}_{k}(t)=\mathcal{M}_{k} \sin \left(2 \pi\left(f_{k}+f_{k+1}\right) t\right) .
$$

The value of $\mathcal{M}_{k}$ is empirical and borrowing from [29] is chosen to be $\mathcal{M}_{k}=5.5 * M_{k}$, with $M_{k}>0$ the magnitude of the spectrum of the $k$ th frequency component.

R3. Identify two cases depending on the physical values of the highest frequency components $f_{1}$ and $f_{2}$, and their associated amplitudes $M_{1}$ and $M_{2}$ :

\footnotetext{
${ }^{1}$ Without loss of generality, we consider $1 \mathrm{~Hz}$ as the boundary between the low frequency and high frequency signals. Therefore we consider signals with frequency components lower or equal to $1 \mathrm{~Hz}$ as low frequency signals.
}

Case 1: If one of the following conditions hold:

a) $f_{1} \leq 1$ and $M_{1}<R_{21} M_{2}$;

b) $f_{1}>1$ and $f_{1} \leq R_{1} f_{2}$;

c) $f_{1}>1$ and $R_{1} f_{2}<f_{1}<R_{2} f_{2}$ and $M_{1}<$ $R_{22} M_{2}$

d) $f_{1}>1$ and $f_{1} \geq R_{2} f_{2}$ and $M_{1}<R_{23} M_{2}$

where $R_{21}=1.1, R_{1}=1.5, R_{2}=2, R_{22}=2$ and $R_{23}=0.5$, then

1.1. Use only the first masking signal

$$
\operatorname{mask}_{1}(t)=\mathcal{M}_{1} \sin \left(2 \pi\left(f_{1}+f_{2}\right) t\right)
$$

for the whole process.

1.2. Construct two signals $x^{+}(t)=x(t)+\operatorname{mask}_{1}(t)$ and $x^{-}(t)=x(t)-\operatorname{mask}_{1}(t)$. Perform EMD on each signal following steps $\mathrm{S} 1$ to $\mathrm{S} 3$ from the standard EMD to obtain all IMFs from each of them, i.e., $c_{i}^{+}(t)$ and $c_{i}^{-}(t), i=1,2, \ldots, n$ and also the residue $r_{n}^{+}(t)$ and $r_{n}^{-}(t)$.

1.3. The IMFs and the residue of the signal $x(t)$ are

$$
\begin{aligned}
c_{i}(t) & =\frac{\left(c_{i}^{+}(t)+c_{i}^{-}(t)\right)}{2}, \quad i=1,2, \ldots, n \\
r_{n}(t) & =\frac{\left(r_{n}^{+}(t)+r_{n}^{-}(t)\right)}{2} .
\end{aligned}
$$

1.4. The total reconstructed signal $\tilde{x}(t)$ is

$$
\tilde{x}(t)=\sum_{i=1}^{n} c_{i}(t)+r_{n}(t) .
$$

Case 2: If other than the conditions a) to d) hold, then 2.1. Use all the constructed masking signals (16).

2.2. Construct two signals $x^{+}(t)=x(t)+\operatorname{mask}_{1}(t)$ and $x^{-}(t)=x(t)-\operatorname{mask}_{1}(t)$. Perform EMD to each signal to obtain the first IMF only from each one, i.e., $c_{1}^{+}(t)$ and $c_{1}^{-}(t)$. The first IMF of $x(t)$ is

$$
c_{1}(t)=\frac{\left(c_{1}^{+}(t)+c_{1}^{-}(t)\right)}{2} .
$$

2.3. Obtain the residue $r_{1}(t)=x(t)-c_{1}(t)$.

2.4. Use the next masking signal, perform steps 2.2 and 2.3 iteratively using each masking signal while replacing $x(t)$ with the residue obtained at each iteration, until $n-1$ IMFs containing the frequency components $f_{2}, f_{3}, \ldots, f_{n}$ are extracted. The final residue $r_{n}(t)$ will contain the remainder.

2.5. Compute the final residue, $r_{n}(t)=x(t)-c_{n}(t)$. 2.6. If the residue $r_{n}(t)$ is above the threshold value of error tolerance, then repeat Step S2 of the sifting process presented in Section II-A on $r_{n}(t)$ to obtain the next IMF and new residue.

2.7. The total reconstructed signal $\tilde{x}(t)$ is

$$
\tilde{x}(t)=\sum_{i=1}^{n} c_{i}(t)+r_{n}(t) .
$$

Remark 3.1: In the complete R-EMD algorithm, we combine the proposed masking algorithm, referred to as Case 1, and the masking algorithm from [29], referred to as Case 2. As can 
be seen clearly from the required conditions stated in the algorithm, Case 1 is active during the "extreme conditions" when the frequency components are low $\left(f_{1} \leq 1\right)$ or when the first two highest frequency components are very close to each other. On the other hand Case 1 takes care of the excluded conditions, particularly to decompose high frequency signals. Therefore, the whole process of R-EMD can handle the decomposition for a large sets of signals both with high and low frequency components.

Moreover, the values of the parameters $R_{1}, R_{2}, R_{21}, R_{22}$ and $R_{23}$ in Case 1 are chosen based on the relation between the frequency as well as the amplitude of the first two highest frequency components of the composite signals. In this paper, the values are chosen to suit the application for signals that contain interarea oscillation. The choice helps classifying signals that satisfies the three reasons given at the beginning of this section. Although they are not optimal, the chosen combination yields effective decomposition for a large set of signals. In general, seeing the EMD algorithm as a filtering process, we can think of the parameters as filter gains that are possible to tune if necessary.

The R-EMD algorithm gives different procedures for dealing with high frequency signals and low frequency signals. The main difference is in the way the masking signals are utilized. For Case 2, we use as many masking signals as the number of frequencies (or ideally the number of frequencies minus one) we want to extract from the signal, and we subtract the effect of each masking signal at every sifting stage, after each IMF is obtained. On the other hand, for Case 1, we use only the first masking signal, constructed from the first two highest frequency components peaking on the Fourier spectrum and let the masking signal stay until the end of the decomposition process. The effect of this masking signal is then automatically removed from the signal through the use of formula (18).

Remark 3.2: The use of only one masking signal constructed using the two highest frequency components of the spectrum in Case 1 is justified, since it satisfies the condition of a masking frequency to be higher than the frequency to be masked. The significant advantage of this algorithm is that it preserves well the magnitude of the signal components, which is not the case for other algorithms as the decomposition often fails. Hence, not only that the instantaneous frequency of the IMFs obtained using the R-EMD algorithm is more meaningful, but also we can obtain a quite good estimation of the instantaneous magnitude of the IMFs.

\section{EMD Method With Energy-Based Masking Technique}

In the previous subsection we use FFT to construct the masking signals, which implies that to some extent we rely of FFT to separate the frequency components of the composite signals. In this section, we extend this approach by deriving the masking signal directly from the EMD. This results in an automated procedure in which the masking procedure is embedded in the EMD decomposition.

Drawing on Case 1 in Section III-B and the notion of instantaneous mean frequency in [27], an alternative approach to determining an appropriate masking signal is suggested, relaxing the dependence on Fourier analysis for detecting the frequency components of the signal. The algorithm, called A-EMD, is summarized as follows.

A1. Perform the standard EMD algorithm on the original signal $x(t)$ to obtain the IMFs. Use only the first IMF, $c_{1}(t)$, which is expected to contain the highest frequency component of the signal, $f_{\max }$, but may also contain mode mixing with other lower frequency components. Perform Hilbert transform on $c_{1}(t)$ to obtain its instantaneous frequency $f_{1}(t)$ and instantaneous magnitude $A_{1}(t)$.

A2. In the spirit of Hilbert analysis, compute the energy weighted mean of $f_{1}(t)$ over $L$ samples, i.e.,

$$
\bar{f}=\frac{\sum_{i=1}^{L} A_{1}(i) f_{1}^{2}(i)}{\sum_{i=1}^{L} A_{1}(i) f_{1}(i)} .
$$

A3. Observe Case 1 from $\mathrm{R} 3$, then replace step 1.1 with the following.

\subsection{Construct the masking signal}

$$
\operatorname{mask}_{1}(t)=\mathcal{M}_{1} \sin (2 \pi(m \bar{f}) t)
$$

where $\mathcal{M}_{1}=\max _{i=1, \cdots, L} A_{1}(i)$ and $m>1$. The rest follows the steps given in the R-EMD algorithm.

Remark 3.3: If the maximum frequency of the composite signal, $f_{\text {max }}$, is lower than $1 \mathrm{~Hz}$, it is common to choose $m=2$ since a higher value of $m$ may cause the masking signal ineffective as its frequency, $m \bar{f}$, would be much higher than $f_{\max }$. Comparing with [27], where the masking signal is computed as $\operatorname{mask}_{1}(t)=a_{0} \sin \left(2 \pi\left(\bar{f} / f_{s}\right) t\right)$, the parameter $m$ replaces the parameter $f_{s}$, the sampling rate. Moreover, we introduced $\mathcal{M}_{1}=\max _{i=1, \cdots, L} A_{1}(i)$ for analytical choice of $a_{0}$ in [27]. To complete the formulation of the method, an efficient algorithm to extract instantaneous attributes is now explored based on the use of a local Hilbert transform.

\section{Convolution-Based Local Hilbert Transform}

Existing approaches to the calculation of the complex trace (2) are based on the computation of the analytic signal through the Fourier transform. This transform, however, has a global character and suffers from problems such as end effects and leakage. In this section, an alternative approach based on filter banks is proposed that circumvents some of these effects.

Given a signal

$$
x(t)=\sum_{\omega} a(\omega) \cos (\omega t)+b(\omega) \sin (\omega t)
$$

where $a$ and $b$ are the Fourier coefficients

$$
\begin{aligned}
& a(\omega)=\frac{1}{T} \int_{0}^{T} x(t) \cos (\omega t) d t \\
& b(\omega)=\frac{1}{T} \int_{0}^{T} x(t) \sin (\omega t) d t .
\end{aligned}
$$

The transformation to a complex time series is

$$
\begin{aligned}
z(t)= & \sum_{\omega} a(\omega) \cos (\omega t)+b(\omega) \sin (\omega t) \\
& +i[b(\omega) \cos (\omega t)-a(\omega) \sin (\omega t)] \\
= & x(t)+i \hat{x}(t)
\end{aligned}
$$


where $\hat{x}(t)=x_{H}(t)$ is the quadrature function, or the Hilbert transform in (2). The Hilbert transform used in this construction is obtained directly by operating the real component with a convolution filter

$$
\hat{x}(t)=x_{H}(t)=\sum_{l=-M}^{M} x(t-l) h(l)
$$

where $h(\cdot)$ is the convolution filter with unit amplitude response and $90^{\circ}$ phase shift. A simple filter that provides an adequate amplitude response and $(\pi / 2)$ phase response is given by [33] as

$$
h(l)= \begin{cases}\frac{2}{l \pi} \sin ^{2}(\pi l / 2), & l \neq 0 \\ 0, & l=0\end{cases}
$$

where $-M<1<M$. As $M \rightarrow \infty$ the filter (28) yields an exact Hilbert transform. For $M$ finite, the filter introduces ripple effects. To limit these effects, a local Hilbert transform has been developed based on filter banks. As suggested in [34] and [35], the filter banks are developed such that the flatness of the frequency response is maximal for the length of the filter. Defining $z=e^{j \omega}$, a maxflat filter can be defined by

$$
h(z)=\left(\frac{1+z^{-1}}{2}\right)^{2 p} Q_{2 p-2}(z)
$$

where $p$ is the number that determine the zeros at $\omega=\pi$, and $Q$ is chosen such that $h(z)$ is halfband. The filter $h(z)$ is shifted in frequency by $(\pi / 2)$.

\section{APPLiCATIONS}

To further illustrate the usefulness of the method, we consider both synthetic data and data from transient stability simulations. For comparison, the system response is analyzed using various algorithms described in previous subsections.

\section{A. Application to a Synthetic Signal}

As a first example, we examine again the synthetic signal (15) that we have used in Section III-A. In order to verify the accuracy and generality of the present method we examine again the synthetic signal 14 in Section II-A with the two-fold objective of evaluating the ability of the method to extract modal components and assessing its generality to deal with nonlinear signals. Previous studies have shown that conventional analysis fails to separate the individual modal making physical interpretation difficult. We focus first on the decomposing capability of the method. Then, we test the ability of the refined technique to deal with nonlinear/nonstationary signals.

1) Decomposing Capability Test: Fig. 4 shows the first three IMFs extracted following the R-EMD algorithm, while Fig. 5 shows the spectra of the first and the second IMFs. For error analysis, $\mathrm{IMF}_{1}$ and $\mathrm{IMF}_{2}$ are also compared with the corresponding components of the composite signal (15) which are plotted as the dashed lines background. Overall, the improved method provides superior temporal resolution. The frequencies identified from Hilbert analysis are 0.8 and $0.5 \mathrm{~Hz}$, which are in

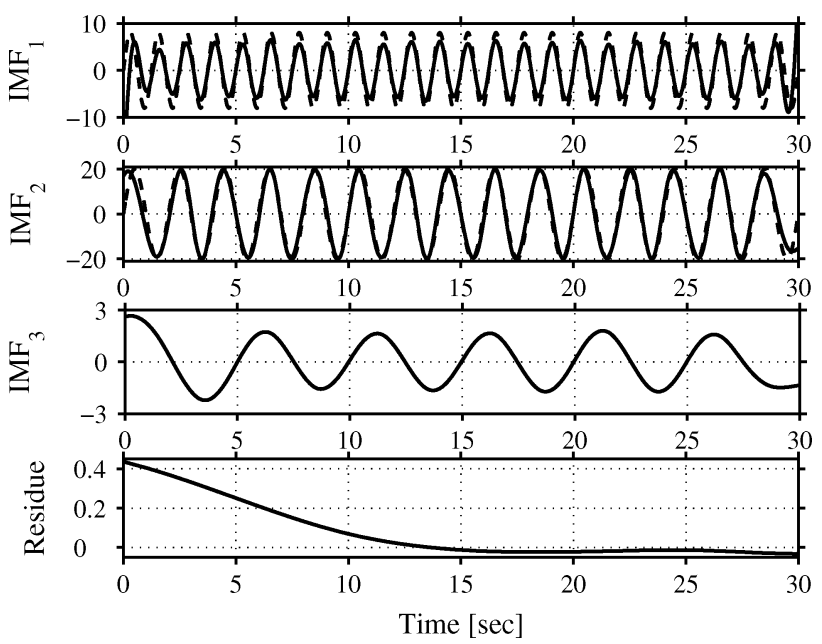

Fig. 4. IMFs of the signal (15) obtained using the R-EMD. The dashed lines are the individual components making up the signal (15). (a)
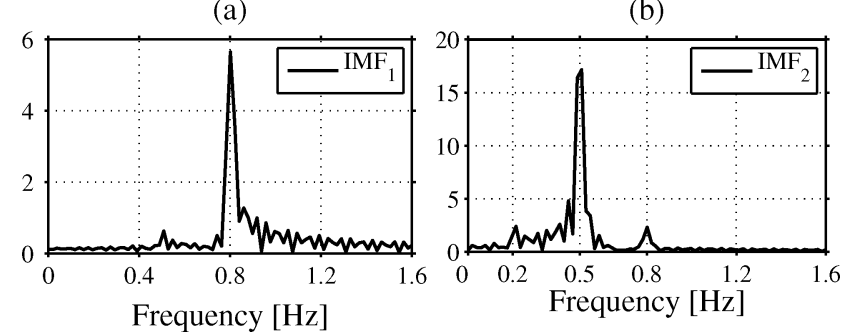

Fig. 5. Fourier spectra of the first and second IMFs of signal (15) with R-EMD

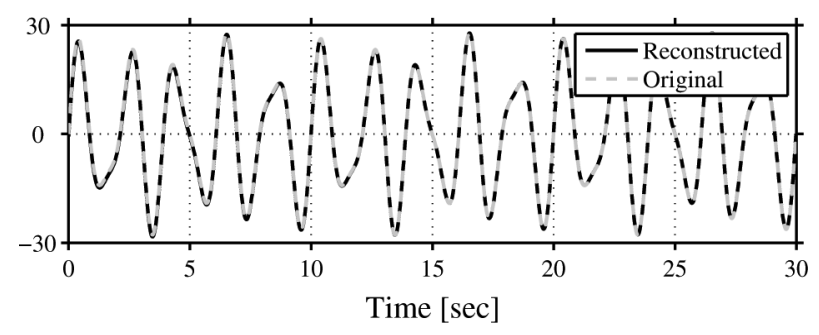

Fig. 6. Reconstruction of signal (15) from IMFs obtained using R-EMD.

agreement with the expected behavior. In addition, comparing Figs. 4 and 5 we can clearly see that although R-EMD relies on FFT to estimate the frequency components of the signal, in fact R-EMD provides correction that yields more accurate information of the amplitude of each components. Fig. 6 shows the correctness of the whole decomposition results and the completeness of the algorithm.

Fig. 7 (a) and (b) shows the instantaneous frequency of $\mathrm{IMF}_{1}$ and $\mathrm{IMF}_{2}$, respectively, which show the frequency components of the composite signal. This figure also compares the instantaneous frequency obtained utilizing the command hilbert in Matlab, with the convolution approach proposed in Section III-D, where the latter is seen to reduce end effects.

The following conclusions can be drawn from this analysis. First, that R-EMD achieves a higher temporal resolution than the standard methods. Second, the convolution based Hilbert transformer provides smoother transformation of the signal by 
(a)

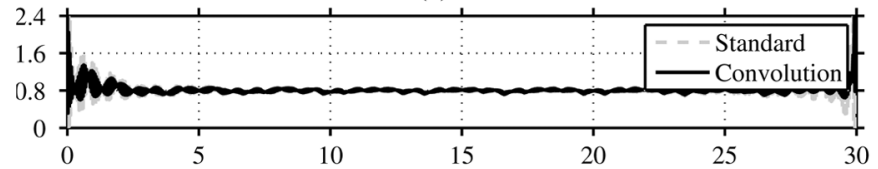

(b)

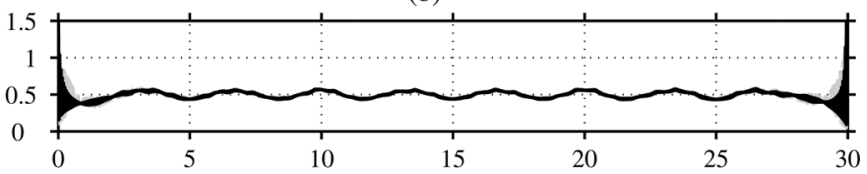

(c)

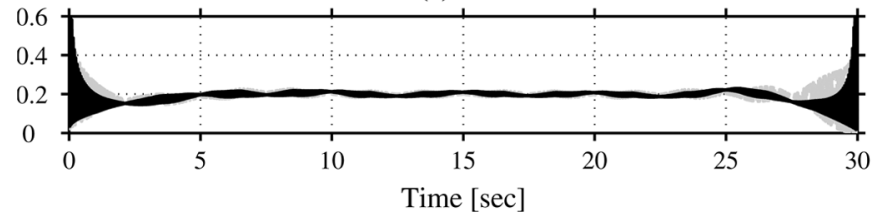

Fig. 7. Instantaneous frequency of the IMFs of the synthetic signal (15). (a) Frequency IMF1. (b) Frequency IMF2. (c) Frequency IMF3.

reducing end effects. The combined application of these approaches results in a more accurate physical characterization of temporal behavior of the signal.

We have also tested the energy based A-EMD algorithm on signal (15). However we do not include the simulation plots in this paper as they are very similar to the results from the R-EMD algorithm. We will show the application of the A-EMD in the next example.

2) Reliability To Handle Nonlinear/Nonstationary Signals: As a second example to assess the ability of the method to treat general signals, a nonlinear and nonstationay version of the signal (15) was examined by clipping the modal components at specific time intervals (see [36] for more details). This gives raise to both harmonic components and nonstationary behavior. Comparison of the decomposition results with the distorted 0.8 and $0.5 \mathrm{~Hz}$ components in Fig. 8 shows that the A-EMD technique effectively deals with abrupt changes in the signals. Although we only show two IMFs, the decomposition actually yields three additional IMFs of negligible magnitude. Table I compares the modes identified using the refined HHT in the paper with modes identified using Prony analysis. For the R-RMD modes, average values are shown.

Although we only show two IMFs in Fig. 8, this decomposition actually yields another three insignificant IMFs plus a residue (as shown partly in Fig. 4). However, feeding the distorted signal using a standard Prony analysis tool (we have used the BPA/PNNL Ringdown Analysis Tool) for comparison, we obtain more elements of the signals. Moreover, applying the instantaneous damping computation formula (14), we obtain the comparison between HHT with R-EMD and Prony analysis as provided in Table I. It is shown that the estimated damping ratio $(\zeta)$ obtained using HHT is more accurate than with the Prony analysis as the damping of the first two IMFs are approximately zero. As suggested in Fig. 8, Hilbert analysis interprets nonlinearity/nonstationarity in terms of frequency and amplitude modulation. As observed in column 3 of Table I, the HHT produces three additional frequency components in addition to the main frequency components, which correspond to the amplitude modulation from the two main frequency components

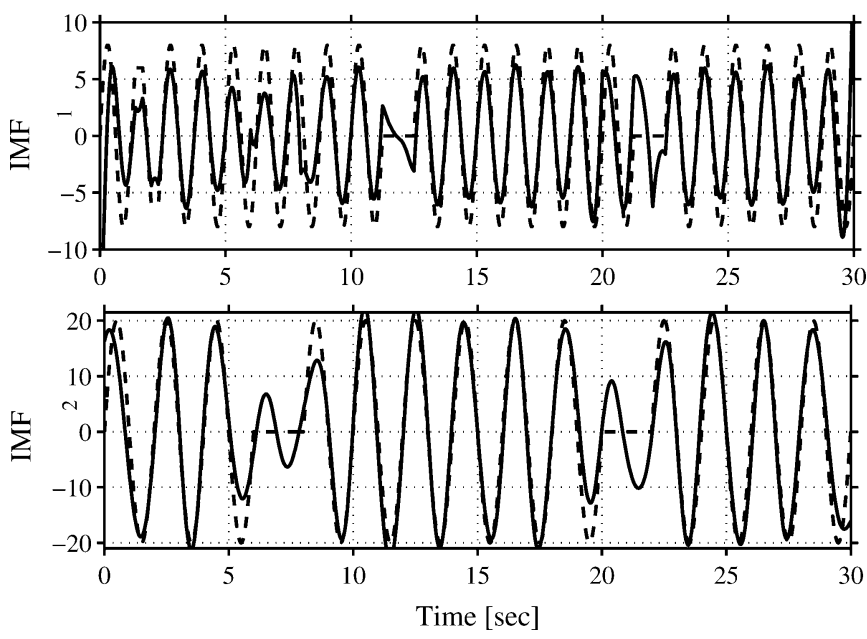

Fig. 8. First two IMFs of the distorted signal (15) obtained using the R-EMD (distorted components are plotted as dashed line background).

TABLE I

COMPARISON OF R-EMD RESULTS AND PRONY ANALYSIS RESULTS

\begin{tabular}{|c|c|c|c|c|c|c|}
\hline \multicolumn{2}{|c|}{ Dist. Signal } & \multicolumn{2}{|c|}{ HHT (mean values) } & \multicolumn{3}{|c|}{ Prony } \\
\hline $\begin{array}{c}\text { Freq. } \\
\mathrm{Hz}\end{array}$ & $\zeta$ & $\begin{array}{c}\text { Freq. } \\
\mathrm{Hz}\end{array}$ & $\zeta$ & $\begin{array}{c}\text { Freq. } \\
\mathrm{Hz}\end{array}$ & $\zeta$ & R. Energy \\
\hline 0.8 & 0 & 0.8016 & 0.0013 & 0.8006 & 0.0030 & 0.1496 \\
\hline 0.5 & 0 & 0.5083 & 0.0001 & 0.4994 & 0.0048 & 1.0000 \\
\hline & & 0.2151 & -0.0238 & 0.3390 & 0.0409 & 0.0375 \\
\hline & & 0.1369 & 0.0106 & 0.1866 & 0.1980 & 0.0301 \\
\hline & & 0.0833 & 0.0055 & 0.0921 & 0.9536 & 0.3269 \\
\hline & & & & 6.3298 & 0.0615 & 0.3180 \\
\hline
\end{tabular}

and the decomposition inaccuracy. This is in marked contrast to Prony analysis in column 5 of Table I, which necessitates a large number of modal components to accommodate nonlinear/nonstationary features.

Moreover, as discussed in our analysis of power system data, Hilbert analysis naturally identifies the time intervals in which the signal is nearly stationary. This may, in fact, help in identifying time intervals in which Prony (Fourier) analysis are meaningful.

Up to this point, we have verified that our proposed algorithms provide a better alternative implementation of HHT in certain applications. We now explore the ability of the method to analyze power system data.

\section{B. Application to Simulated Data}

To verify the proposed method further, we consider simulation data from transient stability simulations of a complex system. Fig. 9 depicts a simplified diagram of the test system showing the study area and major interfaces selected for study [22].

Several simulation studies have been conducted to assess the applicability of the proposed technique to analyze composite oscillations resulting from major system disturbances. In these studies, the southeastern-central interface TEC-TOP was chosen for analysis because this corridor has a dominant participation in three major interarea modes. Fig. 10 shows the power flow response of a key transmission line interconnection, to the loss of Laguna Verde unit \#1. This particular contingency 


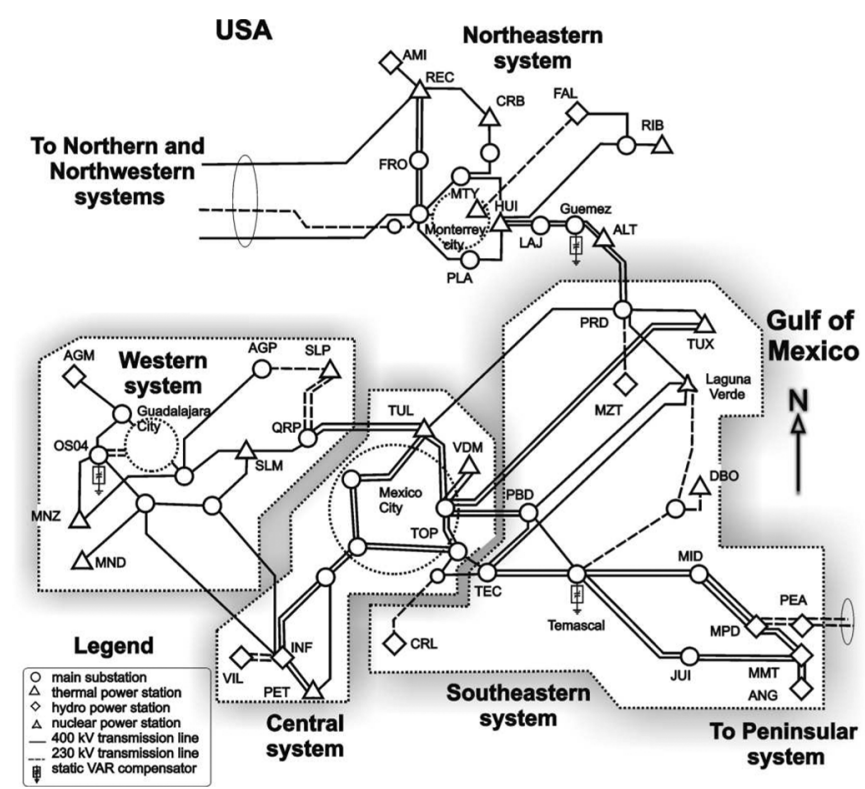

Fig. 9. Simplified geographical scheme of the Mexican interconnected power system.

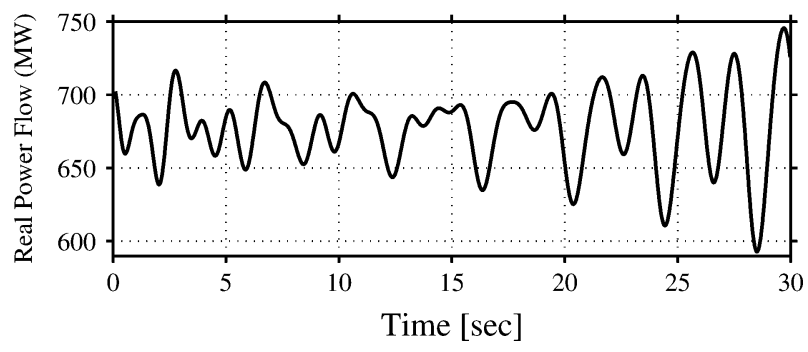

Fig. 10. Tie-line oscillations following the loss of Laguna Verde unit \#1.

results in undamped oscillations involving three major interarea modes at $0.25,0.50$, and $0.78 \mathrm{~Hz}$.

Using the R-EMD method, we decompose the signal into four nonstationary temporal signals and a trend. The IMFs derived using the R-EMD are shown in Fig. 11. For comparison, the IMFs derived from the same signal using the conventional approach are shown in Fig. 12. This is the same information as what has been reported in [22, Fig. 5].

Comparison between Figs. 11 and 12 shows that R-EMD successfully decompose the signal into its essential mono-frequency components. Effectively, the method allows for the nonstationary behavior of the signal to be analyzed into separate temporal scales. In sharp contrast with this, standard EMD results in intermodulation and nonlinear behavior that makes it difficult to extract the physical interpretation of the basic modal properties.

Moreover, it can be seen from Fig. 13 that the R-EMD algorithm accurately extracts the three dominant frequencies as we can see the value of the instantaneous frequency of each IMF is quite constant through out the time. This has shown that the decomposition works well. Figs. 13 and 14 also show that the computation of the instantaneous frequency and the instantaneous magnitude using the convolution based Hilbert transform
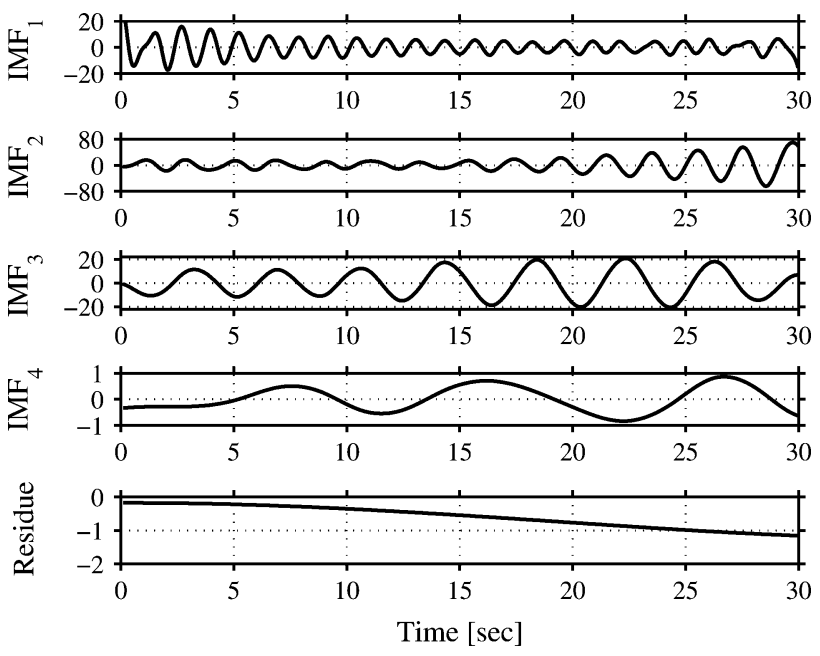

Fig. 11. IMFs obtained using R-EMD algorithm.
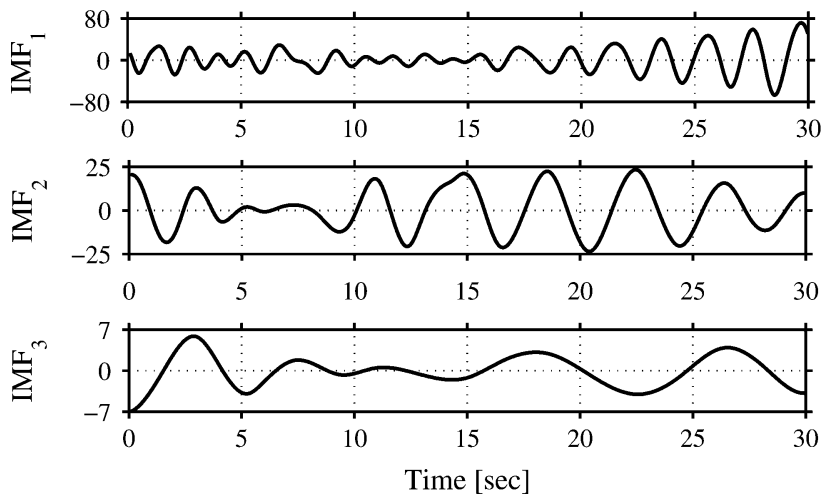

Fig. 12. First three IMFs obtained using standard EMD algorithm.

(a)

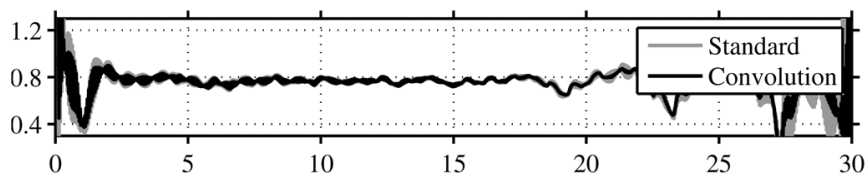

(b)

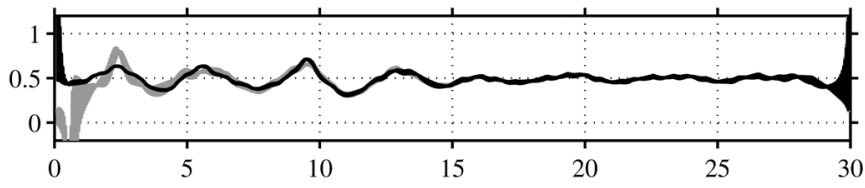

(c)

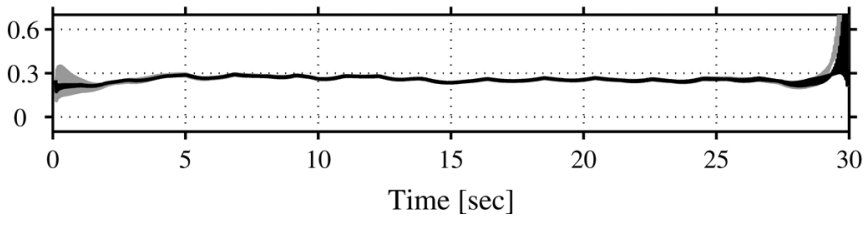

Fig. 13. Instantaneous frequency of the IMFs showing the frequency of the interarea oscillation. (a) Frequency IMF1. (b) Frequency IMF2. (c) Frequency IMF3.

reduces the edge effect that appears strongly when using the standard Hilbert transform. 
(a)

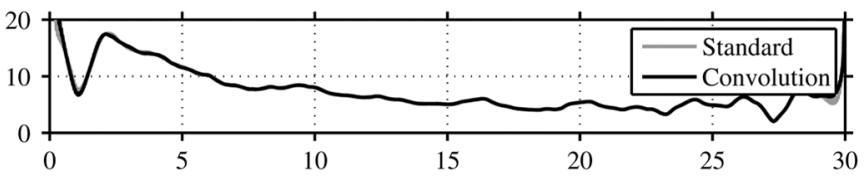

(b)

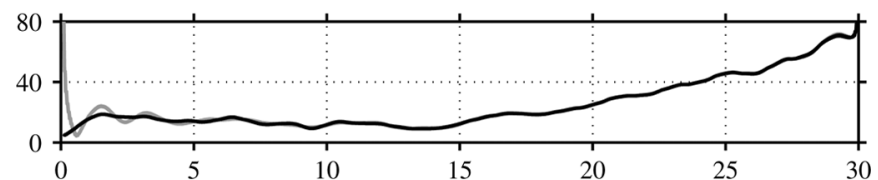

(c)

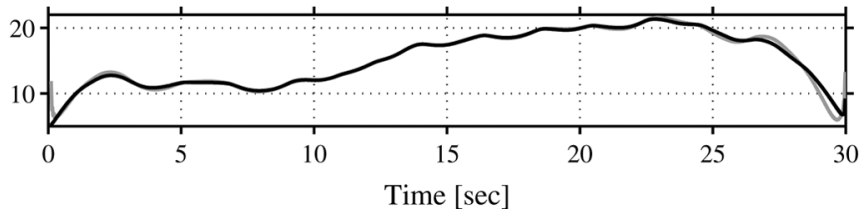

Fig. 14. Instantaneous magnitude of the IMFs showing the growth of each component. (a) Magnitude IMF1. (b) Magnitude IMF2. (c) Magnitude IMF3.

The frequency component of the interarea modes obtained from the power signal in this study (see Fig. 13) are, respectively, $0.7625,0.4888$, and $0.2542 \mathrm{~Hz}$; these modes coincide very well with detailed eigenvalue analysis of the system [22].

Another advantage of this approach over other existing methods is that modal damping can be determined more accurately since the individual (modal) components are isolated and extracted. This issue is discussed with more details in [37].

In order to demonstrate that Hilbert analysis correctly identifies system behavior, we also show that the damping ratio listed in [22, Table III] for the frequency components $0.7625,0.4888$, and $0.2247 \mathrm{~Hz}$, which are, respectively, $0.0173,-0.0209$, and -0.0351 , matches the trend of magnitude of each frequency component. As we can observe from Figs. 11 and 14, the 0.7625 $\mathrm{Hz}$ component is decreasing, the $0.4888 \mathrm{~Hz}$ is increasing, and the $0.2542 \mathrm{~Hz}$ is also increasing.

Figs. 15 and 16 are the corresponding IMFs and instantaneous frequency computed using the A-EMD method. Comparison of Figs. 15 with 11 and Figs. 16 with 13 shows that the two methods give results that show good agreement. In both cases, the local Hilbert transform is found to reduce the end effects.

The numerical implementation of the masking technique in A-EMD deserves some comments. In the actual implementation of the algorithm it may be tempting to question why we are using (24) with the term $m \bar{f}$ instead of using the maximum value of the instantaneous frequency $f_{1}(t)$ of the first IMF that is logically the maximum frequency component of the signal and replace $m \bar{f}$ with $m f_{1, \max }$ where $1<m<2$. Extensive numerical simulations, as illustrated by Fig. 17, show that spikes in the instantaneous frequency computation that appears due to the inaccuracy of the first decomposition with the standard EMD (before the masking signal is constructed) will give a wrong information of the value of the maximum frequency component that leads to the frequency of the constructed masking signal too high hence ineffective. Clearly, the use of $\bar{f}$ in (24) helps in filtering the fictitious variations which in turn results in improved system characterization.

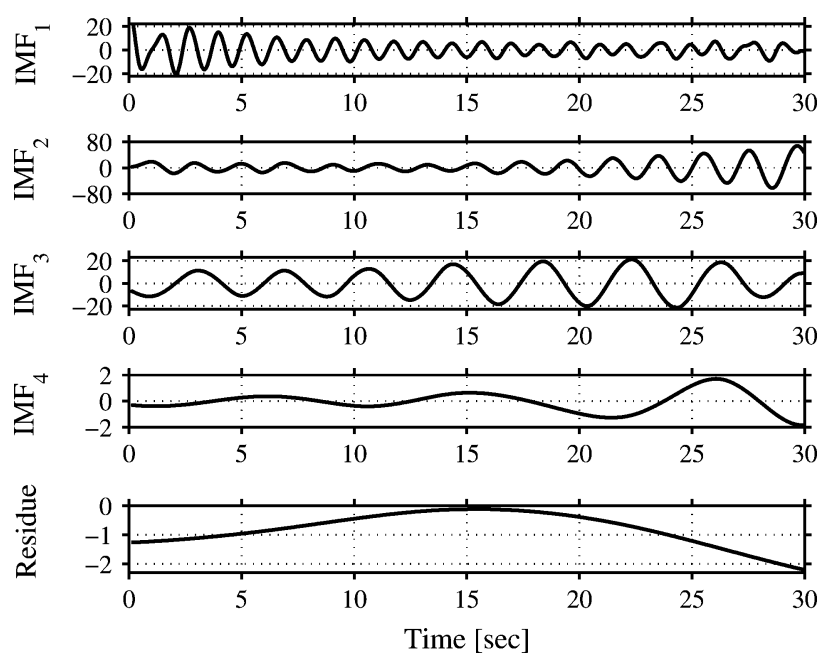

Fig. 15. IMFs obtained using A-EMD algorithm.

(a)

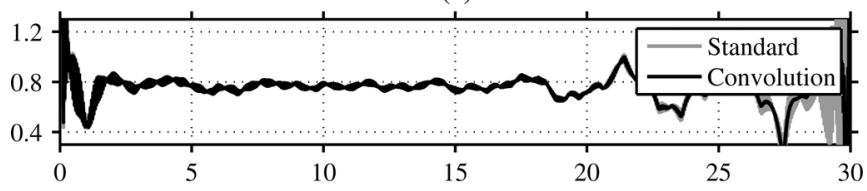

(b)

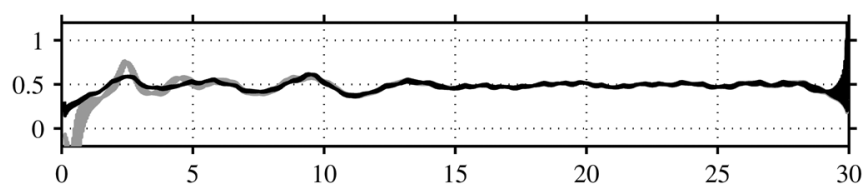

(c)

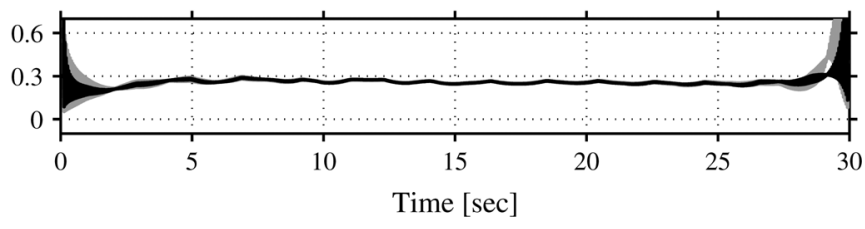

Fig. 16. Instantaneous frequency of the IMFs obtained using A-EMD algorithm. (a) Frequency IMF1. (b) Frequency IMF2. (c) Frequency IMF3.

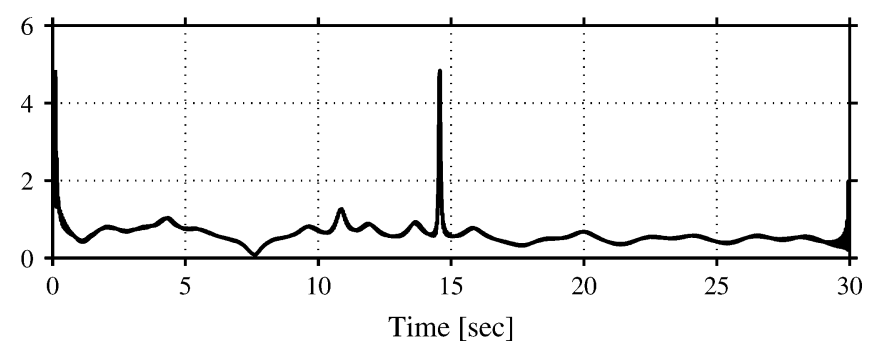

Fig. 17. Instantaneous frequency of the first IMF obtained using the standard EMD algorithm.

To complete our study, we also make a comparison between HHT with A-EMD and Prony. The result is presented in Table II. It can be observed that the results obtained using Prony involve some ambiguities as can be seen for the components 0.4915 and $0.5276 \mathrm{~Hz}$ as well as the components 0.2494 and $0.2758 \mathrm{~Hz}$ as they are coming as pairs. Although the relative energy of the pairing components are significantly different, it tells us that the 
TABLE II

COMPARISON BETWEEN HHT WITH A-EMD AND PRONY FOR INTERAREA MODES ANALYSIS

\begin{tabular}{|c|c|c|c|c|c|}
\hline Modes & \multicolumn{2}{|c|}{ HHT (mean values) } & \multicolumn{3}{|c|}{ Prony } \\
\hline $\begin{array}{c}\text { Freq. } \\
\mathrm{Hz}\end{array}$ & $\begin{array}{c}\text { Freq. } \\
\mathrm{Hz}\end{array}$ & Damping & $\begin{array}{c}\text { Freq. } \\
\mathrm{Hz}\end{array}$ & Damping & R. Energy \\
\hline 0.78 & 0.7625 & 0.010 & 0.7678 & 0.0119 & 0.1032 \\
\hline 0.5 & 0.4888 & -0.010 & $\begin{array}{l}0.4915 \\
0.5276\end{array}$ & $\begin{array}{r}-0.0271 \\
0.0250\end{array}$ & $\begin{array}{l}1.0000 \\
0.0489\end{array}$ \\
\hline 0.25 & 0.2542 & -0.007 & 0.2494 & -0.0257 & 0.2542 \\
\hline & - & - & 0.2758 & 0.0257 & 0.0622 \\
\hline & 0.0978 & -0.030 & 1.1983 & 0.1548 & \\
\hline & & & 0.8635 & 0.0420 & \\
\hline
\end{tabular}

damping information does not show the real damping ratio of the true component 0.5 and $0.25 \mathrm{~Hz}$, respectively. If the components of the monitored signal are not known, this creates confusion in interpreting the results. On the contrary, HHT with A-EMD gives more reliable and consistent results for the decomposition and the damping computation.

These findings are very useful for monitoring and analysis of the interarea oscillation for power system. It has simplified the analysis, as in this way the instantaneous frequency and instantaneous damping of the interarea oscillation can be seen clearly and directly from visual observation, which is very useful when engineers have to make quick decision to take action in urgent situations.

\section{CONCLUSION}

In this paper, a nonstationary data-based, refined approach for characterizing temporal behavior based on the Hilbert-Huang transform has been proposed. The method allows automated extraction and characterization of temporal modal behavior with no prior assumptions on the governing processes driving the oscillations and can be applied to a wide-variety of signals found in power system oscillatory processes.

Simulation results have shown that the proposed algorithms improve visualization of complex oscillations involving multitime scale behavior. The theory can be explored more in several important ways. Further refinement to the technique is possible, including the optimal design of filters and the computation of more general masking techniques. The study also raises a number of challenging issues that will be addressed in future stages of this work. The application of the developed techniques to measured data is being actively investigated by the authors and will be presented in a future publication.

\section{ACKNOWLEDGMENT}

The authors would like to thank J. Thambirajah and N. Thornhill from the Chemical Engineering Department, Imperial College London, for useful comments and discussion that significantly improve the quality of this paper.

\section{REFERENCES}

[1] J. F. Hauer and J. G. DeSteese, A Tutorial on Detection and Characterization of Special Behavior in Large Electric Power Systems Pacific Northwest Nat. Lab., Rep. PNNL-14655. Richland, WA, 2004.

[2] Analysis and Control of Power System Oscillations, CIGRE Technical Brochure No. 111, 1996, C. 38.01.07.
[3] J. F. Hauer, C. J. Demeure, and L. L. Scharf, "Initial results in Prony analysis of power system response signals," IEEE Trans. Power Syst., vol. 5, no. 1, pp. 80-89, Feb. 1990.

[4] J. F. Hauer, "Application of Prony analysis to the determination of modal content and equivalent models for measured power system response," IEEE Trans. Power Syst., vol. 6, no. 3, pp. 1062-1068, Aug. 1991.

[5] D. N. Kosterev, C. W. Taylor, and W. A. Mittelstadt, "Model validation for the August 10, 1996 WSCC system outage," IEEE Trans. Power Syst., vol. 14, no. 3, pp. 967-979, Aug. 1999.

[6] A. R. Messina, V. Vittal, D. Ruiz-Vega, and G. Enríquez-Harper, "Interpretation and visualization of wide-area PMU measurements using Hilbert analysis," IEEE Trans. Power Syst., vol. 21, no. 4, pp. 1763-1771, Nov. 2006.

[7] G. Rogers, Power System Oscillations. Boston, MA: Kluwer, 2000.

[8] D. J. Trudnowski, M. K. Donnelly, and J. F. Hauer, "A procedure for oscillatory parameter identification," IEEE Trans. Power Syst., vol. 9, no. 4, pp. 2049-2055, Nov. 1994.

[9] P. Kundur, Power System Stability and Control. New York: McGrawHill, 1994.

[10] P. W. Sauer and M. A. Pai, Power System Dynamics and Stability. Englewood Cliffs, NJ: Prentice-Hall, 1998.

[11] J. J. Sanchez-Gasca and J. Chow, "Performance comparison of three identification methods for the analysis of electromechanical oscillations," IEEE Trans. Power Syst., vol. 14, no. 3, pp. 995-1002, Aug. 1999.

[12] I. Kamwa and L. Gerin-Lajoie, "State-space system identification toward MIMO models for modal analysis and optimization of bulk power systems," IEEE Trans. Power Syst., vol. 15, no. 1, pp. 326-335, Feb. 2000.

[13] D. J. Trudnowski, J. M. Johnson, and J. F. Hauer, "Making Prony analysis more accurate using multiple signals," IEEE Trans. Power Syst. vol. 14, no. 1, pp. 226-231, Feb. 1999.

[14] D. R. Ostojic and G. T. Heydt, "Transient stability assessment by pattern recognition in the frequency domain," IEEE Trans. Power Syst., vol. 6, no. 1, pp. 231-237, Feb. 1991.

[15] D. R. Ostojic, "Spectral monitoring of power system dynamic performances," IEEE Trans. Power Syst., vol. 8, no. 2, pp. 445-451, May 1993.

[16] R. W. Wies, J. W. Pierre, and D. J. Trudnowski, "Use of ARMA block processing for estimating stationary low-frequency electromechanical modes of power systems," IEEE Trans. Power Syst., vol. 18, no. 1, pp. 167-173, Feb. 2003.

[17] D. J. Trudnowski, J. W. Pierre, N. Zhou, J. F. Hauer, and M. Parashar, "Performance of three mode-meter block-processing algorithms for automated dynamic stability assessment," IEEE Trans. Power Syst., vol. 23, no. 2, pp. 680-690, May 2008.

[18] R. W. Wies, A. Balasubramanian, and J. W. Pierre, "Combining least mean adaptive filter and auto regressive block processing techniques for estimating the low-frequency electromechanical modes in power systems," in Proc. IEEE Power Eng. Soc. General Meeting, Montréal, QC, Canada, 2006.

[19] N. Zhou, J. W. Pierre, and J. F. Hauer, "Initial results in power system identification from injected probing signals using a subspace method," IEEE Trans. Power Syst., vol. 21, no. 3, pp. 1296-1302, Aug. 2006.

[20] N. E. Huang, Z. Shen, S. R. Long, M. L. Wu, H. H. Shih, Q. Zheng, N. C. Y. C. C. Tung, and H. H. Liu, "The empirical mode decomposition and the Hilbert spectrum for nonlinear and nonstationary time series analysis," Proc. R. Soc. London, vol. 454, pp. 903-995, 1998.

[21] T. J. Browne, V. Vittal, G. T. Heydt, and A. R. Messina, "A comparative assessment of two techniques for modal identification form power system measurements," IEEE Trans. Power Syst., vol. 23, no. 3, pp. 1408-1415, Aug. 2008

[22] A. R. Messina and V. Vittal, "Nonlinear, non-stationary analysis of interarea oscillations via Hilbert spectral analysis," IEEE Trans. Power Syst., vol. 21, no. 3, pp. 1234-1241, Aug. 2006.

[23] J. C. Echeverria, J. Crowe, M. Woolfson, and B. R. Hayes-Gill, "Application of empirical mode decomposition to heart rate variability analysis," Med. Biol. Eng. Comput., vol. 39, no. 4, pp. 471-479, 2001.

[24] B. M. Battista, C. Knapp, T. McGee, and V. Goebel, "Application of the empirical mode decomposition and Hilbert-Huang transform to seismic reflection data," Geophysics, vol. 72, no. 2, pp. H29-H37, 2007.

[25] M. A. Andrade, A. R. Messina, C. A. Rivera, and D. Olguin, "Identification of instantaneous attributes of torsional shaft signals using the Hilbert transform," IEEE Trans. Power Syst., vol. 19, no. 3, pp. 1422-1429, Aug. 2004. 
[26] Z. Wu and N. E. Huang, "A study of the characteristics of the white noise using the empirical mode decomposition method," Proc. R. Soc. London A, vol. 460, pp. 1597-1611, 2004.

[27] R. Deering and J. F. Kaiser, "The use of a masking signal to improve empirical mode decomposition," in Proc. IEEE Int. Conf. Acoustic, Speech, and Signal Processing (ICASSP'05), 2005, vol. 4, pp. 485-488.

[28] R. Deering, "Final-scale analysis of speech using empirical mode decomposition: Insight and applications," Ph.D. dissertation, Duke Univ., Durham, NC, 2006.

[29] N. Senroy and S. Suryanarayanan, "Two techniques to enhance empirical mode decomposition for power quality applications," in Proc. IEEE Power Eng. Soc. General Meeting, Tampa, FL, 2007, pp. 1-6.

[30] N. Senroy, S. Suryanarayanan, and P. F. Ribeiro, "An improved Hilbert-Huang method for analysis of time-varying waveforms in power quality," IEEE Trans. Power Syst., vol. 22, no. 4, pp. 1843-1850, Nov. 2007.

[31] R. R. Zhang, L. vanDemark, J. Liang, and Y. Hu, "On estimate site damping with soil non-linearity from earthqueake recordings," Int. J. Nonlin. Mech., vol. 39, pp. 1501-1517, 2004.

[32] G. Rilling, Empirical Mode Decomposition, Matlab Codes With Examples, Jan. 2008. [Online]. Available: http://perso.ens-lyon.fr/patrick. flandrin/emd.html.

[33] W. B. White and S. E. Pazan, "Hindcast/forcast of ENSO events based upon the redistribution of observed and model heat content in the western tropical Pacific, 1964-86," Control Phys. Oceanography, vol. 17, pp. 264-280, 1987.

[34] R. L. C. Spaendonck, F. C. A. Fernandes, R. G. Baraniuk, and J. T. Fokkema, "Local Hilbert transformation for seismic attributes," in Proc. EAEG 64th Conf. Tech. Exhib., 2002.

[35] G. Strang and T. Nguyen, Wavelets and Filter Banks. Reading, MA: Wellesley-Cambridge Press, 1997.

[36] D. S. Laila, M. Larsson, B. C. Pal, and P. Korba, "Nonlinear damping computation and envelope detection using Hilbert transform and its application to power systems wide area monitoring," in IEEE Power and Energy Soc. General Meeting, 2009, to be published.

[37] D. S. Laila, A. R. Messina, and B. C. Pal, "Variants of Hilbert-Huang transform with applications to power systems oscillatory dynamics," in Analysis of Nonlinear and Non-stationary Inter-area Oscillations: A Time-Frequency Perspective, A. R. Messina, Ed. New York: Springer, 2009.

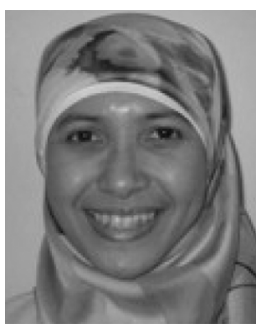

Dina Shona Laila (S'94, M'03) was born in Jakarta, Indonesia, on January 28, 1973. She received the B.Eng. and M.Eng. degrees, both cum laude, from the Institute of Technology Bandung (ITB), Bandung, Indonesia, in 1995 and 1998, respectively, and the Ph.D. degree in control engineering from the University of Melbourne, Victoria, Australia, in 2003.

She was a research student in Araki Laboratory, Electrical Engineering Department, Kyoto University, Kyoto, Japan (1996-1998). She has been a research associate at the Control and Power Group, Imperial College London (2003-2006 and 2007-present) and with the Institute for Design and Control of Mechatronics Systems, Johannes Kepler Univerisity Linz (1996-1997). Her main research interest is design and analysis of nonlinear control systems, as well as nonlinear identification, with various applications including mechatronic systems and power systems.

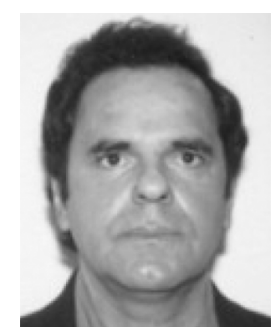

Arturo Roman Messina (M'85-SM'05) received the M.Sc. degree (with honors) in electrical engineering from the National Polytechnic Institute of Mexico, Mexico City, in 1987, and the Ph.D. degree from Imperial College, London, U.K., in 1991.

Since 1997, he hasbeen a Professor at the Center for Research and Advanced Studies (Cinvestav), Guadalajara, Mexico.

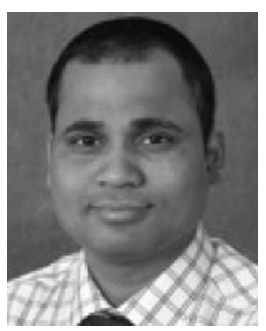

Bikash C. Pal (M'00-SM'02) received the B.E.E. (with honors) degree from Jadavpur University, Calcutta, India, the M.E. degree from the Indian Institute of Science, Bangalore, India, and the Ph.D. degree from Imperial College London, London, U.K., in 1990, 1992, and 1999, respectively, all in electrical engineering.

Currently, he is a Reader in the Department of Electrical and Electronic Engineering, Imperial College London. His current research interests include state estimation, power system dynamics, and flexible ac transmission system controllers. 